\title{
Diagnosis and management of Recurrent Herpetiform Stomatitis and Behçet Syndrome Like Recurrent Aphthous Stomatitis herpetiform type
}

\author{
Endah Ayu Tri Wulandari*, Gus Permana Subita** \\ *Resident Department of Oral Medicine Faculty of Dentistry Universitas Indonesia \\ **Department of Oral Medicine Faculty of Dentistry Universitas Indonesia
}

ABSTRACT

Recurrent Aphthous Stomatitis (RAS) is a common inflammatory condition of the oral mucosa. The aetiology of RAS remains unclear, yet there are several predisposing factors which could be involved in the onset of the lesion. The herpetiform type of RAS appeared to be similar to recurrent oral Herpes Simplex infection and also could be part of Behçet Syndrome. This case report discussed a patient suffering from herpetiform type of RAS with its clinical appearance resembling recurrent oral Herpes Simplex infection and Behçet syndrome. Initial treatment was undertaken based on the empirical treatment, yet the respond was not satisfactory. Then, laboratory tests were undertaken, including complete blood count, total population of T lymphocyte, B lymphocyte, T helper, T supressor, NK cells, $\mathrm{T}$ helper/T supressor ratio, C3, C4, IgG, IgA, and IgM. These tests showed that there were immune and hematinic deficiency condition. Nevertheles, the clinical appearance, laboratory findings and consultation did not support the diagnosis of recurrent oral Herpes Simplex infection and Behçet Syndrome, thus, enhancing the definite diagnosis of the herpetiform type of RAS with immune and hematinic deficiency as the underlying condition. Based on the definite diagnosis, treatment plan was then revised to target the underlying condition.

Key words: Recurrent Aphthous Stomatitis, Behçet Syndrome, recurrent oral Herpes Simplex virus infection

\section{INTRODUCTION}

Recurrent Aphthous Stomatitis (RAS) is an inflammatory condition of oral mucosa which is characterized by shallow ulceration and recurrent paint with several days to several month intervals. ${ }^{1 .}$ ${ }^{4}$ This condition is found in $20 \%$ of the population ${ }^{2,3}$, with about $60-85 \%$ of RAS are experienced initially before 30 years of age and predominantly found in women. ${ }^{3}$ Recurrent Aphthous Stomatitis has three forms, i.e. RAS mayor, minor and herpetiformis. ${ }^{1-4}$ In general, patients experience one of the three types of RAS. However, it is possible that a patient experiences 2 types of RAS at the same time or experience clinical appearance change from one type of RAS to another type of RAS along the time. ${ }^{3,4}$ RAS etiology is not known yet. There are several predisposition factors of RAS including hereditary/genetic factors, hormonal change, hypersensitivity towards certain food, drug, 
stress, local trauma, bacterial/viral infection and hematinic/nutrition deficiency. ${ }^{1-4}$

Behçet Syndrome is a multisystem abnormality with mucocutaneous, ocular, arthritic, vascular and central nervous system involvement predominantly found in male with a clinical appearance of genital ulceration, eye lesion, skin lesion, and positive pathergic test. ${ }^{2,4}$ ${ }^{6}$ Aphthous ulceration appears in $99 \%$ of Behçet Syndrome and is the first symptom seen in $67 \%$ of the cases. In Behçet Syndrome, RAS in minor, mayor or herpetiformis type can be seen. . $^{2,-6}$

Talking about Herpes, there is a kind of Herpes Simplex viral infection that is the most frequent causes of vesicle development in the oral cavity. ${ }^{7}$ Primary HSV-1 infection can be asymptomatic but also may create prodromal symptoms in the form of fever, myalgia and malaise 1 or 2 days before the lesion appear. ${ }^{7}$ The thin walled vesicle will easily broken and leave a round shallow ulcer with a very small size. However, the small ulcers may integrate and create a big ulcer. ${ }^{7,8}$ Latent type 1 Herpes Simplex virus will stay in trigeminal nervous ganglion. ${ }^{9}$ Fever, menstruation, trauma and immunosuppression are several factors that may reactivate HSV-1. ${ }^{8,9}$ Recurrent Intraoral Herpes (RIH) lesion is very similar to RAS herpetiformis. Several things differentiate both lesions. In RIH lesion, the lesion is always started with a vesicle when the RAS herpetiformis does not. In addition, RIH lesions are found in thick keratinous mucosa while RAS herpetiformis lesions are found in non keratinous mucosa. ${ }^{9}$ So, definitly those kinds of diseases can be different.

In this case study, one case of oral ulceration case accompanied by ulceration in the genital and eye complaint that was initially diagnosed as Recurrent Herpetic Stomatitis and then diagnosed as Behçet Syndrome with a final diagnosis is RAS herpetiformis with underlying hematinic deficiency is presented. A comprehensive management is needed to manage a complex RAS case.

\section{CASE REPORT}

A 41 year old female was referred from the Dermatology and Venereal Disease Clinic of Cipto Mangunkusumo Central Hospital/RSCM with a working diagnosis of complex Aphthous and a comparative diagnosis of Behçet Syndrome. The patient complained of oral ulcers outbreak that spread in her oral cavity since 7 days before, which followed a fever attack. The ulcers were followed by aphthous-like lesion in the genital since 5 days before and red eyes with stinging feeling were experienced 4 days before.

The patient had had recurrent aphthous since the age of 25 years old with a rare frequency and in a small number. However, the outbreak of aphthous became more often in the last 1 year and this time it was felt as the most severe. The aphthous-like outbreak in genital and eye complaint were never experienced before. There is a recurrent aphthous history in her family.

Clinical examination showed a large number of ulcerated lesions spread in the oral cavity. The size was $1-10 \mathrm{~mm}$ in the upper and lower labial mucosa, palatum durum mucosa and molle, right and left buccal mucosa, gingiva, mouth floor, and the dorsal and lateral area of the tongue (see Fig. 1). The working diagnosis made in the first visit was Herpetic Stomatitis with a comparative diagnosis of RAS herpetiformis or Behçet Syndrome. The management applied at that time included tetracycline mouthwash and dyphenhydramine $\mathrm{HCl}$ that were used alternately, zinc-containing multivitamin, and complete peripheral blood (CPB) test as well as antibody serologic test for HSV- 1 and HSV-2.

The CPB showed a normocytic normochromal anemia while the antibody serological test for HSV showed an anti HSV-1 IgG (+) with a value of 3.30. In the second visit, the patient felt that the aphthous condition was better than before and the ulcers in the genital were already healed. However, since the eye complaint became more severe, the patient was referred to the Ophthalmologist Clinic of Cipto Mangunkusumo Central Hospital/RSCM with a result that showed an immunological marginal keratitis. In the clinical examination of the oral cavity, there were still ulcers that spread in the oral cavity in a smaller number and size. During this visit, the patient was instructed to continue for using the tetracycline and dyphenhydramine $\mathrm{HCl}$ mouthwash alternately and she was given immunomodulator containing echinacea, zinc picolinate and selenium.

On the third visit, the patient received prednison to be used with swish and spit manner, 3 times a day. On the fourth visit, the condition 
seemed better although there were still some ulcers in the mouth but in much less number (see Fig. 2). During this visit, the patient was instructed to use chlorhexidine gluconate $0.2 \%$. Two weeks after this fourth visit, the lesion was resolved and there was no outbreak for the next 4 weeks.

Six weeks after the fourth visit, the patient came with a complaint of Recurrent Aphthous outbreak. The outbreak this time was not accompanied by genital ulcers and eye complaint. In the clinical examination it was found that there were more than 10 ulcerations on the upper labial mucosa, left and right buccal mucosa, palatum molle, the lateral part of tongue and mouth floor (see Fig. 3). During this visit, the patient was again instructed to use prednison mouthwash with swish and spit manner, and to underwent CPB and immunological tests in the form of lymphocyte $T$, lymphocyte B, T helper (Th), T suppressor (Ts), and natural killer (NK) cells total count, T helper/ T suppressor comparison, C3, C4, IgG, IgA, and IgM tests. The CPB showed that the patient experienced microcytic anemia while the immunological tests showed that there was a decreased $T$ cell total number, increased NK cell activation, normal Th/Ts ratio, with a conclusion that there was an immune deficiency accompanied by NK cells activation. In addition, there was also increased level of C3, C4, $\lg A$, and $\lg G$.

TheAphthous came and go without any period of Aphthous free since the fifth visit up to the next 2 months. Genital ulcers and eye complaint never returned. The patient had received multivitamin to control the anemic condition and she was also instructed to improve nutrition intake, which was poor at the first place. On the sixth visit, a stress level measurement using "Perceived Stress Scale" 10 was performed with a result showing that there was a very high stress perception. According to the patient, the economic problem was the main cause of stress. On the seventh visit, clinical examination showed more than 10 ulcers with a size of 2-5 mm in palatum molle mucosa, upper labial mucosa, left and right buccal mucosa, and an erythematous area spread in oral mucosa. The working diagnosis confirmed in this visit was RAS Herpetiformis with anemic background.

On the eighth visit, the patient received systemic prednison with an initial dose of 30 $\mathrm{mg} /$ day that was reduced gradually until $5 \mathrm{mg} /$ day followed by swishing and spitting prednisone 2 times a day for 3 minutes each. In addition, the patient was also given multivitamin to help improving the anemic condition caused by poor nutrition intake. In the clinical examination in the 2 next visits, the oral cavity was free from ulcers (see Fig. 4).

\section{DISCUSSION}

The initial working diagnosis for this case was Recurrent Herpetic Stomatitis based on the clinical examination findings, i.e. groups of ulcers with small size that were spread in all over oral mucosa, both keratinous and non keratinous oral mucosa which were not started by vesicles. The patient also complained of fever during aphthous outbreak although fever is often experienced by patients whose nutrition intake is reduced. The working diagnosis is supported by the results of the antibody serological test for HSV type 1 that shows a high value (3.6 times higher than the normal value). Stoopler et al. ${ }^{7}$ in his article stated that fever, myalgia and malaise are prodormal symptoms in symptomatic primary HSV infection that precedes vesicle formation while Scully et al. ${ }^{8}$ and Scott et al. ${ }^{11}$ stated that fever is one of the factors that trigger HSV reactivation. The prodormal symptoms in recurrent HSV infection include itchy or burning sensation in the place where the vesicle appears which is also the initial sign of virus replication at the sensory nerve end in the dermis or mucosa. ${ }^{11,12}$ The presence of grouped ulcerations with small size in patient that are spread widely in the oral cavity including the palatum durum, is matched with the presentation of HSV type 1 infection in the literatures. ${ }^{13,14}$

The absence of vesicles in patients, both in the clinical examination and in the history taking, may be caused by 2 things: the vesicles are already broken at the time of examination or the patient's lesions are never preceded by vesicles which leads to not enable this ulcerated lesions as a herpetic stomatitis. The result of the antibody serological examination towards HSV according to Stoopler et al. ${ }^{7}$ is important in diagnosing primary HSV infection when there is an increased level 4 times of the normal value or more. However, this examination is useless in diagnosing recurrent HSV infection because only $5 \%$ of the HSV recurrent 
infection patients experience significant increase of antibody level. ${ }^{7}$ Välimaa et al. ${ }^{15}$ in they research found that anti HSV-1 lgG serum level in seropositive subject with Recurrent Herpes Labialis (RHL) is 3.1 times higher than the asymptomatic seropositive subjects. ${ }^{15}$ Seppänen ${ }^{16}$ in his dissertation stated that the presence of anti-HSV-IgG in the serum cannot be used to differentiate HSV infection with other abnormalities with similar appearance. It can be concluded that the patient experienced HSV reactivation.

However, it could not be confirmed whether the lesion in patient's oral cavity is a recurrent HSV infection. To confirm a diagnosis that a doubtful lesion is a recurrent HSV infection lesion or not, supporting examinations such as Tzanck smear, biopsy, immunomorphological assays (direct fluorescent assay and indirect fluorescent assay), viral culture, DNA-HSV examination using Polymerase Chain Reaction (PCR) are preferred. ${ }^{7-}$ 9,11

The Herpetic Stomatitis diagnosis may became RAS herpetiformis based on the followings: recurrent fever for 2 weeks after the beginning of the lesion, ulcerated lesions come and go for 3 consecutive months without any vesicular appearance. Ulcerated lesion became more dominant in non keratinous and thin keratinous mucosa. It could not be ascertained whether the diagnosis was only RAS herpetiformis only or RAS as a part of Behçet Syndrome.

In this case, the Behçet Syndrome working diagnosis was made based on the clinical findings, i.e. recurrent ulceration in the oral cavity accompanied by genital ulcers and eye complaint. This is in line with the international criteria of Behçet Syndrome established in 1990 that include recurrent oral ulceration accompanied by at least two other criteria, i.e. genital recurrent ulcers, eye ulcers (anterior uveitis or posterior uveitis), the presence of cells in vitreous, retinal vasculitis, skin lesion (erythema nodusum, pseudo-vasculitis, papulopustular lesion, acneiform nodules) and positive result for the pathergic test. ${ }^{5}$

The main criterion for Behçet Syndrome diagnosis, was oral recurrent ulceration, while the supporting criterion that was also presented was the genital ulcers, although it was not recurrent. Based on the clinical examination, the eye complaint presented by the patient could not be proven as an abnormality related to Behçet Syndrome. It is suspected that the genital ulceration and marginalis keratitis were separate events from the oral ulceration that occurred at the same time. In this case, histopathological examination was not performed because based on the literature, ulceration in RAS and Behçet Syndrome looks similar, either clinically or histologically. ${ }^{4}$ In this case, the pathergic test was also not performed because based on the literature it is stated that only a minority of the Behçet syndrome cases give positive result to the pathergic test. ${ }^{16}$ The positive result in pathergic test can also be caused by other conditions so that it is not $100 \%$ specific for Behçet Syndrome diagnosis. ${ }^{17}$

Because the findings did not meet the criteria for Behçet Syndrome diagnosis, the possibility is that the patient experienced RAS herpetiformis with other underlying abnormalities such as hematinic deficiency. This is based on the laboratory findings where patients experienced microcytic anemia and immunological deficiency. Based on the anamnesis, it was found that the patient had a poor nutrition intake added by stress factor. Other predisposing factors that can be found in the patient were local trauma in oral cavity and bacterial infection caused by poor oral hygiene and hereditary factor.

Based on CPB examination result, it was found that the patient experienced microcytic anemia which characterizes iron deficiency. This may be a predisposition for genital ulcers and marginalis keratitis. Iron deficiency can suppress cellular immune responses, including the response of lymphocyte, neutrophil, and macrophag that makes the person more prone to infection. ${ }^{18}$ Based on other literatures, iron deficiency may lead to phagocytic ability disorders, reduces lymphocyte stimulation response, reduces NK cells related to decrease interferon production and suppresses delayed cutaneous hypersensitivity responses. ${ }^{19}$

Based on the immunological examination results, it was also found that there was a decrease total number of T cells, increase NK cells, normal Th/Ts ratio with a conclusion that there was an immunological deficiency accompanied by NK cell activation. This may relate to iron deficiency experienced by the patient. In a study, it was found that there were a decrease lymphocyte, $T$ cell and 
Thelper number and reduced $T$ cell proliferative ability in individuals with iron deficiency. ${ }^{10}$ From several studies it was found that RAS improvement was seen after a nutrition substitution therapy, including iron, was applied. ${ }^{21}$

What is interesting in this case is that in iron deficiency, the NK cell number should be reduced while the patient showed an increased NK cell number. The possibility is that the NK cell increase is related to the stress that is experienced by the patient. Based on the results of the stress questionnaire, it was found that the patient experienced a very high level of stress. Stress can cause immune system deficiency where the immune response will disappear after autonomic nervous system activation frequently found in chronic stress case happened. ${ }^{23}$ Based on other literatures, it is revealed that stress can increase CD8 cell division rate leading to increased CD8 cell number and suppresse immune function. ${ }^{22}$ However, with a positive attitude, the ability of the immune system can be increased by increasing NK cell activity. ${ }^{23,24}$

An individual will respond to various types of stressors by rapidly synthesizing heat shock

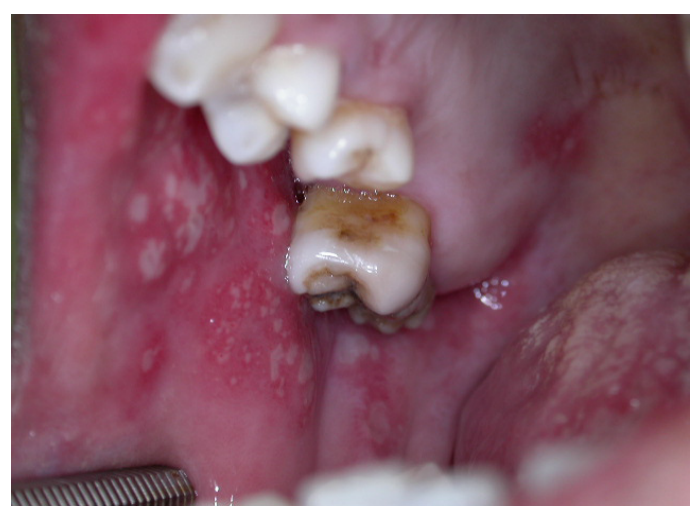

Figure 1. First visit.

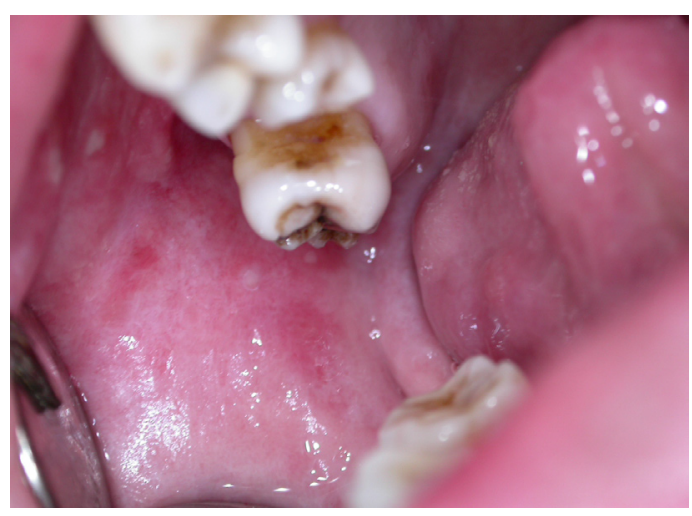

Figure 3. Fifth visit. protein (Hsp) which relates to autoimmune disease initialization. ${ }^{10}$ The heat shock protein itself is actually harmless but the Streptococcus antigen triggers the body to secrete high number of $\gamma \delta T$ cells leading to cross reaction to oral mucosa mitochondrial Hsp induced by stress. ${ }^{10}$ Inflammation found in this case makes tissue ulceration possible due to increase localize antibody attack in tissues. ${ }^{10}$ When an emotional stress occurs, the immune system will be deteriorated leading to infection in oral lesion that gets more severe by the immunosuppression. ${ }^{10}$ The suppressed immune system in the stress condition may also be happened by glucocorticoid release that will block cytokine production leading to immune system obstruction. ${ }^{25} \mathrm{McC}$ artan et al. ${ }^{26}$ in their study on the relationship of stress and RAS stated that nervousness is apparent among RAS patients but the conclusive mechanism is not yet revealed. Moreover, McCartan et al. ${ }^{26}$ suspected that the presence of nervousness among RAS patients will lead to parafunctional habit that will trigger ulceration in vulnerable individuals.

The immunopathogenesis of RAS itself is not fully understood yet. However, it is suspected

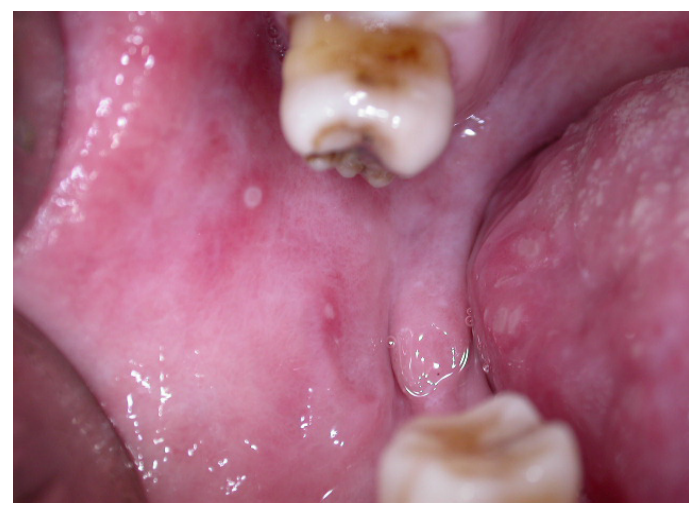

Figure 2. Fourth visit.

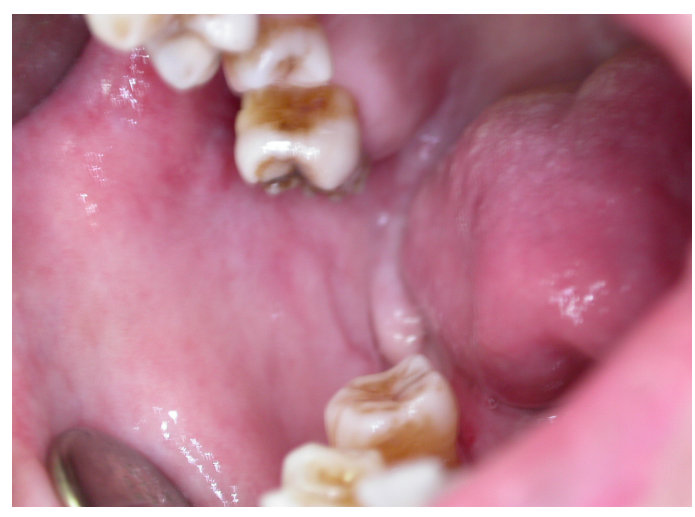

Figure 4. Eighth visit. 
that there is an infiltration of T lymphocyte to the epitheliumasaresponse to the keratinocyte-related antigens. ${ }^{2}$ The keratinocyte death is considered mediated by cytotoxic $T$ cell differentiation that produces Tumor Necrosis Factor-a (TNFa) that induces inflammation due to the adhesion effect of endothelial cells and neutrophil chemotaxis. ${ }^{2,21}$ Another hypothesis is that the presence of oral Streptococcus will directly become pathogenic or stimulate antibody formation leading to cross reaction with keratinocytes. ${ }^{2}$ The cross reaction is possibly caused by the high similarity between the $65 \mathrm{kDa}$ microbial Hsp with $60 \mathrm{kDa}$ human Hsp. ${ }^{2}$ The response mediated by $T$ cells in the cross reaction can induce damages in oral mucosa. ${ }^{3}$

In one study, it is apparent that the peripheral lymphocytes of RAS patients are cytotoxic for oral epithelial cells. ${ }^{3}$ SAR patients experience increased antibody-dependent cellular cytotoxic (ADCC) activity in the early stage of the disease. $^{3,4}$ In the immunological tests, patients show increased level of C3, C4, IgA and IgG, as well as increased NK cell activity. The increased IgG level and NK cell activity in this patient possibly showed an increased ADCC which is a possible ulceration cause. Besides, IgG can also activate a complementary system to lyse the target cells. ${ }^{27}$

In a previous study, an increased NK cell activity during active lesion in oral cavity is seen. A decrease NK cell activity during healing is also found and becomes normal when the lesion dissapear. ${ }^{3,4}$ From the immunological examination results, there seems to be an increased NK cell which raises a possibility that the examination is performed during the active period of RAS. However, the certain role of the NK cells in pathogenesis or RAS healing is still uncertain. ${ }^{4}$

One of the predisposing factors of RAS is hematinic deficiency and the presence of iron deficiency that makes deficiency management necessary by giving iron-containing multivitamin and suggestion to improve nutrition intake. ${ }^{28}$ In one study it is reported that there is a remission after an iron deficiency therapy is applied in RAS patients but other study reported that the effect is only temporary or even none. If the iron deficiency therapy is effective, the hemoglobin $(\mathrm{Hb})$ level will increase about $2 \mathrm{~g} / \mathrm{dl}$ every 3 weeks. ${ }^{29}$ At the time the $\mathrm{Hb}$ level has reached normal value, the therapy must not be stopped abruptly because the iron reservoir kept in the body has not been replaced so that the therapy should be continued for 3 months. ${ }^{29}$

Corticosteroid therapy in the form of topical prednison using swish and spit method does not give good result because the ulceration still comes and goes in a high frequency. This may be affected by low patient adherence in obeying the prednison mouthwash usage instruction. The topical corticosteroid does not give consistent effects on the appearance of new ulcers but can be significantly reduced the severity of the pain caused by ulceration. ${ }^{1}$ Topical corticosteroid has 2 actions, i.e. anti inflammatory action for ulceration development in each stage which reduces the discomfort and a specific blocking action towards lymphocyte $T$ interaction with epithelial cells. ${ }^{2}$ The second action becomes important in topical corticosteroid application because sensitized lymphocytes accumulate before and during the initial stage of oral ulceration. Topical corticosteroid application will give maximum effect when it is applied during the early stage of oral ulceration. ${ }^{2}$ For severe SAR with high recurrent frequency, topical corticosteroid therapy is not enough and a systemic corticosteroid therapy is needed. ${ }^{3,30}$ The prednison systemic corticosteroid is given to reduce inflammation by reducing increased capillary permeability and suppress PMN activity, stabilize lysosomal membrane, reduce lymphocyte and antibody production and decrease inflammation mediators release, monocyte, neutrophil migration and $\mathrm{T}$ cell function. ${ }^{31}$

\section{CONCLUSION}

Recurrent Aphthous Stomatitis is an oral mucosal inflammatory condition with 3 types of clinical appearance with various predisposing factors including hematinic deficiency. Hematinic deficiency can suppress the immune system that supports lesion formation, not only in the oral cavity but also in other parts of the body. This may confuse diagnosis making because it makes the appearance of RAS herpetiformis with anemia background similar to Recurrent Herpetic Stomatitis and Behçet Syndrome. A good investigation is needed to find the true abnormality and the underlying factors. The investigation includes history taking, clinical examination, 
referral to other department, supporting tests, and stress level measurement. A good cooperative effort is needed with other department such as the Dermatology and Venereal Disease Department and Ophthalmology Department in this case.

\section{REFERENCES}

1. Porter S, Scully C. Aphthous ulcers (recurrent). Clin Evid 2004;12:1-2.

2. Field EA, Allan RB. Oral ulcerationaetiopathogenesis, clinical diagnosis and management in the gastrointestinal clinic. Aliment Pharmacol Ther 2003;18:949-62.

3. Natah SS, Konttinen YT, Enattah NS, Ashammakhi N, Sharkey KA, HäyrinenImmonen R. Recurrent Aphthous ulcers today. A review of the growing knowledge. Int J Oral Maxillofac Surg 2004;33:221-34.

4. Natah SS. Recurrent aphthous ulceration. immuno-pathological aspect. Academic dissertation. [cited 2006 Jan 8]. Avalaible from:http://ethesis.helsinki.fi/julkaisut/laa/ $\mathrm{kliin} / \mathrm{vk} / \mathrm{natah} /$ recurrent.pdf.

5. Rabinovich CE, Wagner-Weiner L. Behçet Syndrome. [cited 2006 Des 2]. Avalaible from: http://www.emedicine.com/ped/topic219. htm.

6. Yousefi M, Ferringer T, Lee S, Bang D, Lee ES. Behçet. [cited 2006 Des 2]. Avalaible from: http://www.emedicine.com/derm/topic49. htm.

7. Stoopler ET, Pinto A, DeRossi SS, Sollecito TP. Herpes simplex and varicella-zoster infections : Clinical and laboratory diagnosis. [cited 2007 Feb 17]. Avalaible from:http://www.agd.org.

8. Scully C, Felix DH. Oral medicine-update for the dental practitioner. Aphthous and other common ulcers. British Dent J. 2005;199(5):259-64.

9. Raborn GW, Grace M. Herpes Simplex type 1 orofacial infections. Herpes 1999;6(1):8-11.

10. McNally IM. Recurrent Aphthous Stomatitis and perceived stress. [cited 2006 Jan 5]. Avalaible from:http: / /aphthous-stresstudy.tripod.com.

11. Scott DA, Coulter WA, Biagioni PA, O'Neill H, Lamey PJ. Detection of Herpes Simplex virus type 1 shedding in the oral cavity by polymerase chain reaction and enzyme-linked immunosorbent assay at the prodromal stage of recrudescent herpes labialis. J Pathol Med. 1997;26:305-9.

12. Simmons A. Clinical manifestation and treatment considerations of Herpes Simplex virus infection. J Infectious Disease 2002;186(Suppl 1):S71-7.

13. Tillis TSI, McDowell JD. Differential diagnosis. Is it herpes or aphthous? J Contemp Dent Pract 2002;3(1):1-10.

14. Siegel MA. Diagnosis and management of Recurrent Simplex infections. JADA 2002;133: 1245-9.

15. Välimaa $H$, Waris $M$, Hukkanen V, Blankenvoorde MFJ, Nieuw Amerongen AV, Tenovuo J. Salivary defense factors in Herpes Simplex virus infection. J Dent Res 2002;81(6):416-21.

16. Seppänen $M$. Complement factor C4 and immunoglobulins in recurrent or chronic mucosal infections. Academic Dissertation, 2007 Jan 12. [cited 2007 Feb 17]. Avalaible from:http://ethesis.helsinki.fi/julkaisut.

17. America Behcet's Disease Association. Most common symptoms and signs of Behçet's disease [cited 2006 Mei 3]. Avalaible from: http: / /www. behcets.com/site/pp.asp?c=bhJI JSOCJrH\&b=260548.

18. Oppenheimer SJ. Iron and its relation to immunity and infectious disease. J Nutr 2001;131:616S-5S.

19. Scrimshaw NS, SanGiovanni JP. Synergism of nutrition, infection, and immunity. an overview. Am J Clin Nutr. 1997;66:464S-77S.

20. Ahluwalia N, Sun J, Krause D, Mastro A, Handte G. Immune function is impaired in iron-deficient, homebound, older women. Am J Clin Nutr 2004;79:516-21.

21. Scully C, Gorsky M, Lozada-Nur F. The diagnosis and management of Recurrent Aphthous Stomatitis, A consensus approach. JADA 2003;134:200-7.

22. Koenker H. Stress and immune system. [cited 2006 Jan 5]. Avalaible from:http://www.econ. uinc.edu/hanco/bio/stress.html.

23. Beaton DB. Effects of stress and psychological disorders on the immune system. [cited 2006 Jan 5]. Avalaible from:http://www. personalityresearch.org/paper/beaton.html.

24. Holmes L. How stress affects the immune system. [cited 2006 Jan 5]. Avalaible from: http: / / www. metalhealth.about.com /od/ 
stress/a/stressimmune604.html.

25. Lydyard P, Whelan A, Fanger MW. Immunology. $2^{\text {nd }}$ ed. London: BIOS Scientific Publisher Ltd.; 2004. p. 157-8.

26. McCartan BE, Lamey PL, Wallace AM. Salivary cortisol and anxiety in Recurrent Aphthous Stomatitis. J Pathol Med 1996;25:357-9.

27. Benjamini E, Coico R, Sunshine G. Immunology. A short course. $4^{\text {th }}$ ed. New York: A John Wiley \& Sons Inc.; 2001.

28. Challacombe SJ, Scully C, Keevil B, Lehner T.
Serum ferritin in recurrent oral ulceration. J Pathol 1983;12:290-9.

29. Provan D. Iron deficiency anemia. [cited 2006 Sept 7]. Avalaible from:http://proquest.umi. $\mathrm{com} / \mathrm{pqdweb}$ ?did=625288221 $\mathrm{q}$ sid $=3 \& \mathrm{Fmt}=3 \& \mathrm{c}$ lientld=4562\&RQT=309\&VName=PQD.

30. Scully C. Aphthous ulceration. N Engl J Med. 2006;355:165-72.

31. Medicine and treatment. [cited 2006 Mar 4]. Avalaible from:http://www.behcet.org.il/ medicine\&treatmentE.htm. 